\title{
Illustrating physical principles through comparative feature extraction techniques in optical and digital image processing
}

Ralph Oberly, James Brumfield

Ralph E. Oberly, James O. Brumfield, "Illustrating physical principles through comparative feature extraction techniques in optical and digital image processing," Proc. SPIE 1603, Education in Optics, (1 March 1992); doi: $10.1117 / 12.57878$ 


\title{
Illustrating physical principles through comparative feature extraction techniques in optical and digital image processing
}

\author{
Ralph Oberly and James O. Brumfield \\ College of Science, Marshall University \\ Huntington, WV 25755 USA
}

\begin{abstract}
Facilitation of pattern recognition in machine-vision systems or Thematic Mapper imagery can be used to illustrate physical principles through comparative feature extraction techniques in optical and digital image processing. This is demonstrated utilizing coherent image processing with Fourier transforms incorporating filtering techniques such as convolution and inverse Fourier transform. A parallel digital image processing sequence is applied to the same image. Transparencies of the final product image are optically processed with cross-correlation for comparison of results where applicable
\end{abstract}

\section{Introduction}

In a previous paper ${ }^{1}$ new facilities were discussed with regard to an integrated approach to the teaching of optics. electronics. and computer simulation modeling. This paper is to look at the teaching approach to these discipline areas and the comparison of results between the areas when similar techniques are used. In particular. Fourier transform theory can be used to process images optically or electronically. In the latter case the Fast Fourier Transform (FFT) algorithm is a prime tool for the efficient processing of electronic data representing images obtained from a variety of sources such as remotely sensed earth-resource scenes obtained from satellite or aircraft platforms, or machine-vision images for use in an industrial setting.

The theory of Fourier series and Fourier transforms is available in the literature ${ }^{2-4}$ and will not be repeated here. It is the intent of this paper to emphasize the parallels between optical and electronic image processing using Fourier transforms. The modern digital computer is the tool to perform the transform operation in electronic imagery. The simple lens is the tool in the optical domain. A scene originally recorded using the Thematic Mapper (TM) satellite observation system is used to illustrate some possible results for both systems. The scene has been transformed into a computer compatible digital image for the electronic processing. A black and white negative transparency is made from the digital image for the optical processing. 


\section{Optical and digital image processing equipment and facilities}

The electronic digital image processing capabilities on the Marshall University campus have resulted from the teaching of courses in remote sensing and digital image processing. The remote sensing courses are team taught in a cooperative venture with faculty members from several departments. Within the recently renovated Science Building one room is set aside as a machine-room. (or a laboratory facility). for use in digital image-processing. An adjoining room has terminal space and connections for students and faculty. The laboratory facility contains a MicroVAX II from Digital Equipment Corporation (DEC) that is dedicated to on-site image processing operations. This MicroVAX II supports the operation of an International Imaging Systems (I2S) digital image display system. The MicroVAX II runs on VMS version 5.4 and VAXcluster software via an Ethernet link to the university Computer Center`s VAXcluster. This MicroVAX II manages the I2S image display system using the Land Analysis System (LAS) and the International Mathematics and Statistics Library (IMSL) software system. In addition. Maps Analytical Package System (MAPS) software. Statistical Analysis System (SAS) software. a large variety of DEC software. and other software systems are available to the users on the VAXcluster. The system also provides extensive network telecommunications software for the campus-wide network. state-wide network, and national/international networks such as BITNET. the National Supercomputing Center in Pittsburgh. and EDUnet. (Past work by the authors ${ }^{6-10}$ includes distributed and parallel processing involving the West Virginia Educational Telecomputing Network (WVNET) and/or Hunter College of City University of New York emphasizing digital image processing.)

A second MicroVAX II in the machine-room is also ethernetted to the university's Computer Center VAXcluster that runs on VMS version 5.4. This MicroVAX is used to provide testing. research. and development through computer simulation modeling in the areas of physics, engineering technology, and computer science. This can involve machine-vision. machine sensor system simulations. and expert MRPII + (advanced manufacturing resource planning) through the Robert C. Byrd Institute for Advanced Manufacturing Systems. Eventually this system will network with near real time capabilities through distributive processing via the campus-wide network with other laboratories. the Robert Bird Institute. WVNET and gateways to other national and international network systems.

The Robert C. Byrd Institute for Advanced Manufacturing Systems is located off the main campus in downtown Huntington. It provides the capability for Flexible Computer Integrated Manufacturing Systems (FCIMS) through computer integrated machining cells. robotic systems for materials handling. machine-vision/robot-coupled systems for pallet configuration, and computer networking to facilitate product design through prototyping and process development. These cells include systems from Cincinnati Milacron. Emco-Maier. and Mapix. These facilities provide a real time environment for the research. development. integration and networking of electro-optical sensor systems with machining and process data at disparate locations with final 
testbedding. The combination of classroom/research laboratories on campus and the practical aspects of machine-vision and electro-optical systems in a prototyping industrial setting at the Institute allows students to experience from theory to application.

Referring again to the earlier paper: on the physical facilities. the optics laboratories adjoin the space for image processing. The optical processing is achieved on a conventional optical filtering system ${ }^{11}$ with a coherent source (in this case a $5 \mathrm{~mW} \mathrm{He}-\mathrm{Ne}$ laser), spatial filter, collimating lens. Fourier transform lens. inverse Fourier transform lens, magnifying lens. and a film holder for Polaroid Type 52 film. A 2-meter lathe bed optical bench provides the base for the system.

\section{RESULTS AND CONCLUSIONS}

Students and faculty can work with both the optical and digital image processing systems to gain a greater understanding of filtering processes. Starting with the digital spatial domain, an image can be processed completely without the computer operator ever visually seeing the Fourier transform in the frequency domain. Consequently, the operator possibly never visualizes what happens in the filtering process. In the optical domain high-and low-pass filters are fitted to frequency domain information in such a way that the experimenter must visualize the Fourier transform information. While visualization is inherently a part of the optical process, accuracy is much lower due to less control in filtering frequency domain orders. This is especially true for low spatial frequency objects in the amplitude domain. The optical process offers visualization of the physical principles while the digital process provides greater control of the end result. Both processes used together allow students to gain a full understanding of Fourier filtering theory.

Figure 1 illustrates some of the possibilities for filtering in the digital process. Figure 1a is a digital image obtained from Thematic Mapper (TM) data recorded on $35 \mathrm{~mm}$ monochrome negative film (ISO 400) using the matrix camera of the I'2S system at NASA/Goddard Space Flight Center. The TM scene includes an urban setting. The data are from band one, blue-green. and form an image of 128 lines by 128 pixels. The image was first digitally contrast-stretched using LAS/STRETCH with a distributed scale of plus or minus three standard deviations of the image mean. A two-dimensional Fourier transform was obtained using LAS/FFT2, figure 2a. Figures 1b. 1c. and $1 \mathrm{~d}$ show the results of filtering the Fourier transform with low-, middle- and high-pass filters. respectively. from the LAS package. The actual images result from performing the inverse Fourier transform (LAS/IFFT2) on the filtered transform. With band one being in the blue-green range some image-blurring is expected due to atmospheric scattering. The low-pass image shows a feature that may be a low frequency sinusoidal wave in the radiometrically uncorrected TM data as a result of TM instrumentation/voltage variations under the stated observation conditions, figure $1 \mathrm{~b} .12$ 


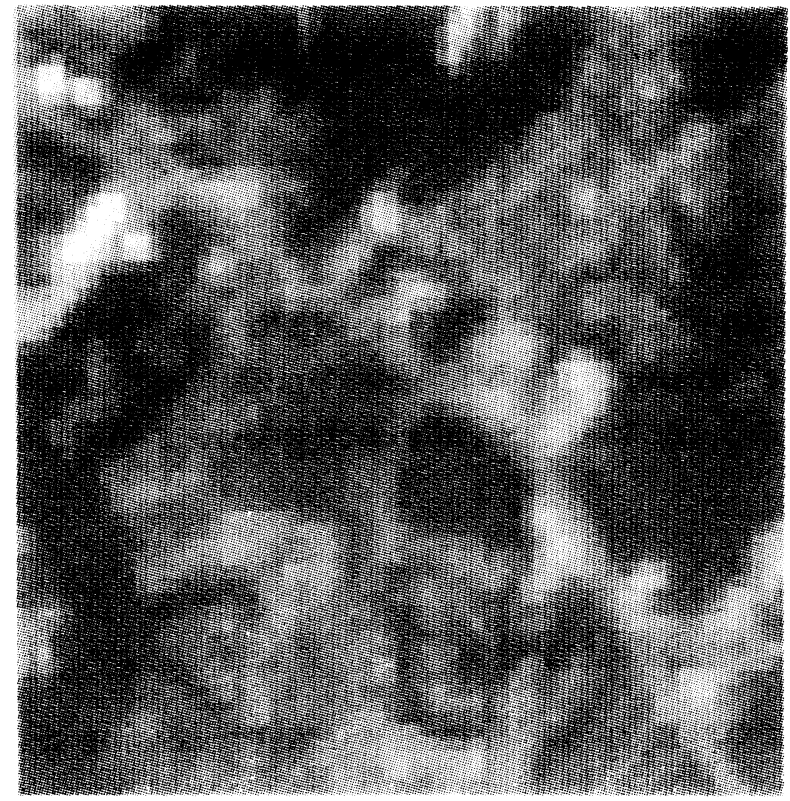

a

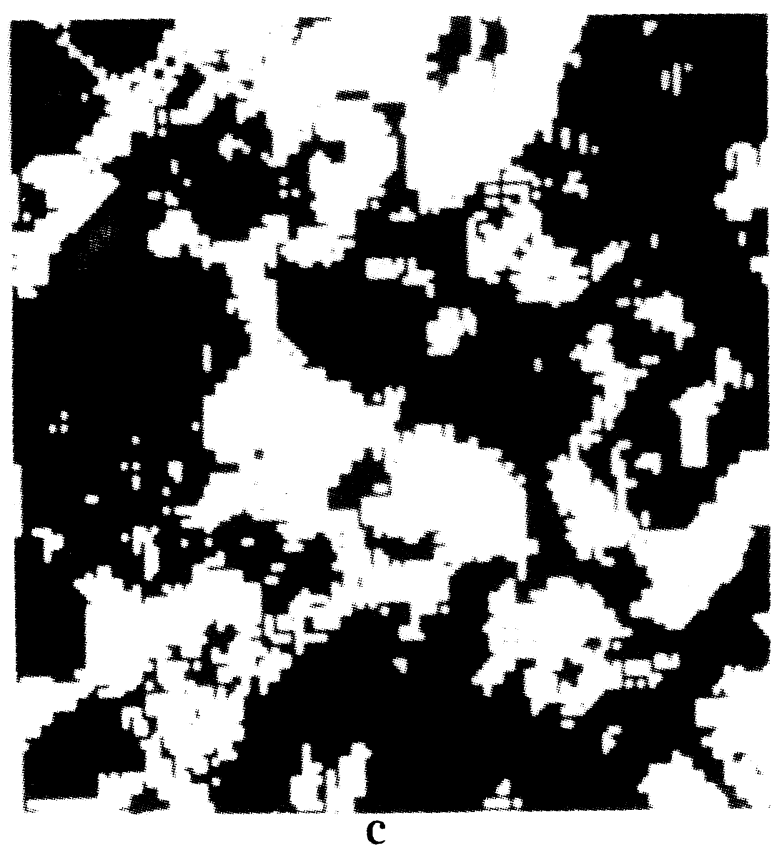

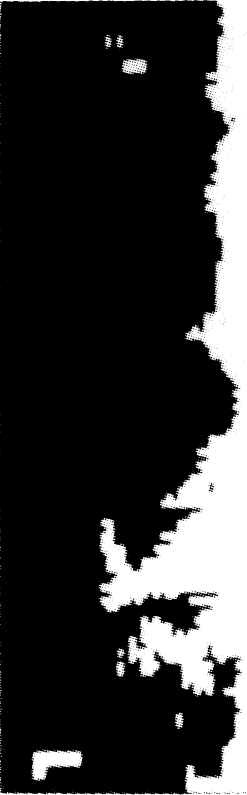

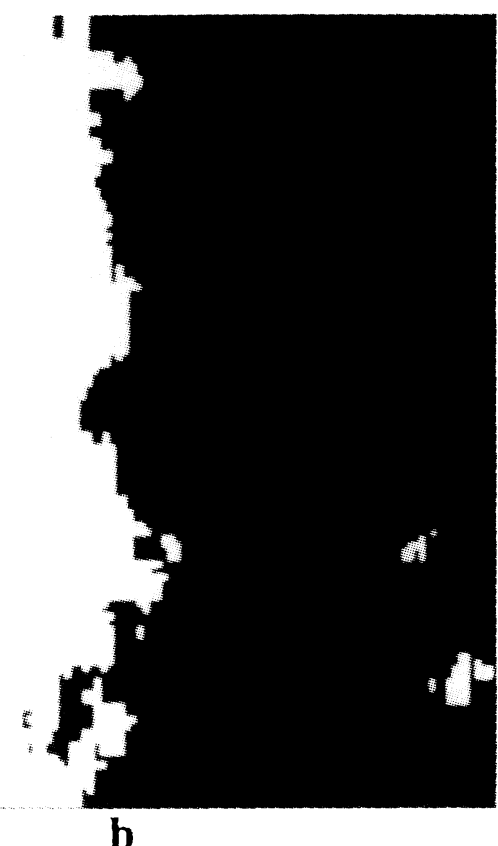

b

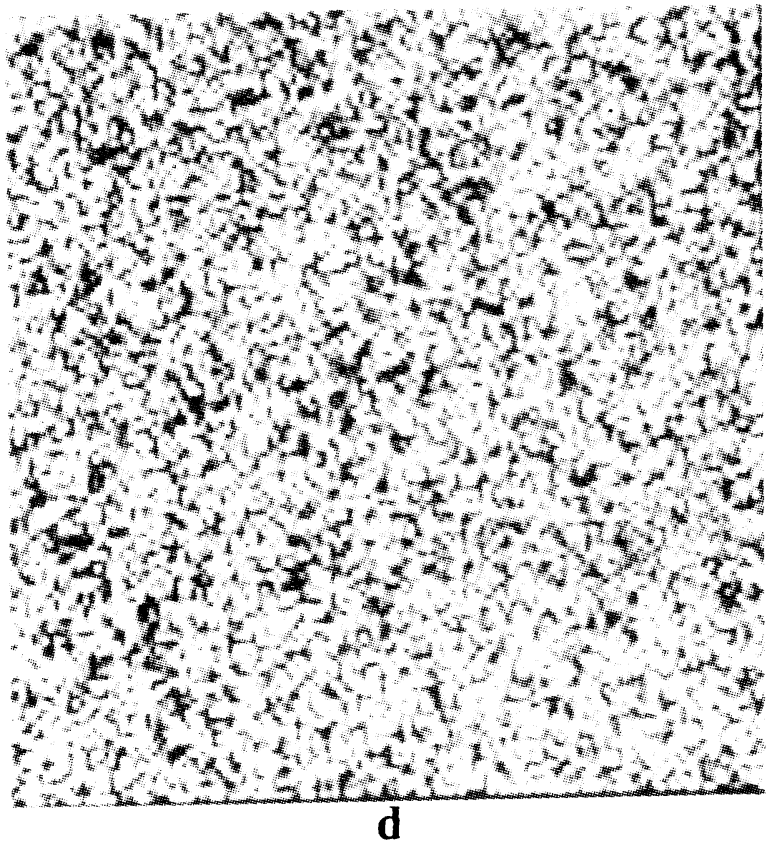

Figure 1

Original Thematic Mapper image (a). image after low-pass filtering (b). image after middle-pass filtering (c), and image after high-pass filtering (d). Images in b. c. and $d$ are spatial domain. 


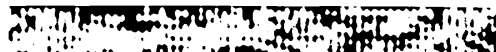

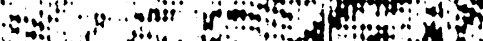
240

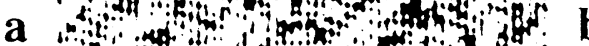
10

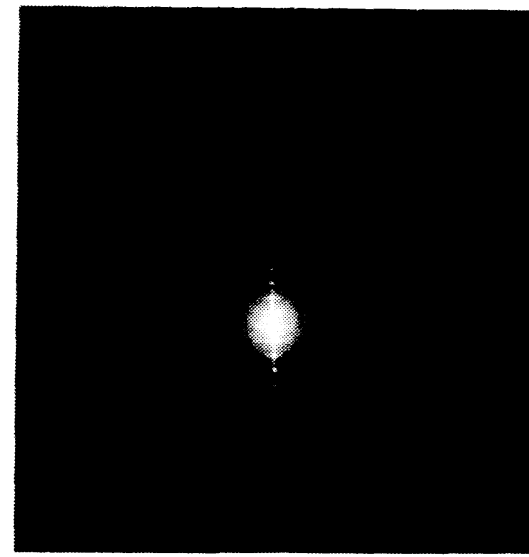

\section{Figure 2}

of

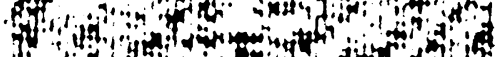
10 Af

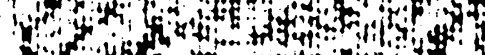

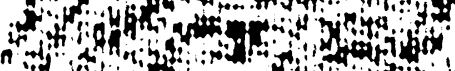

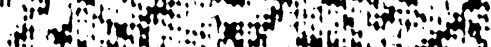
int ${ }^{2}$ riph at end of itg of i......

a

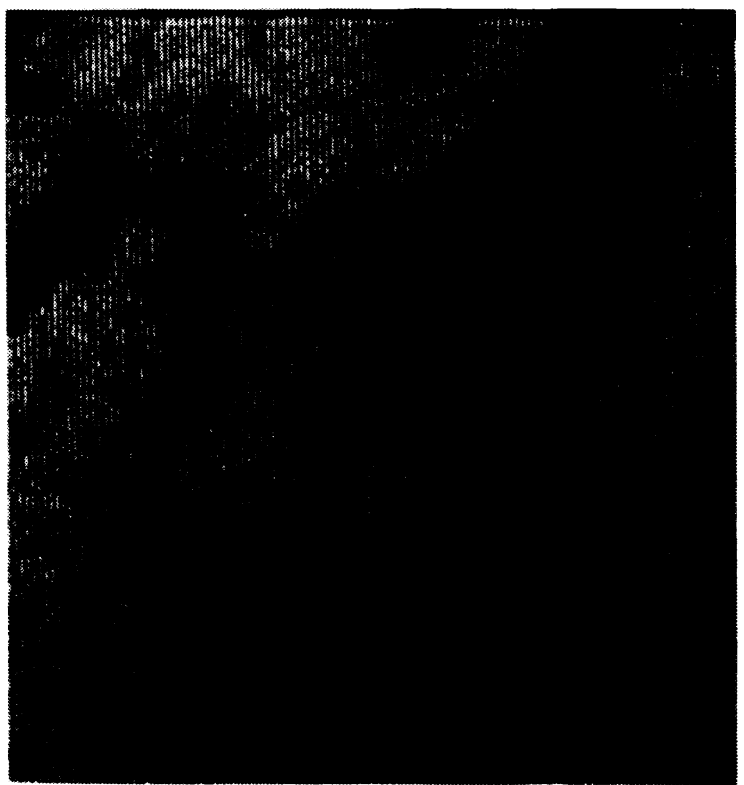

C
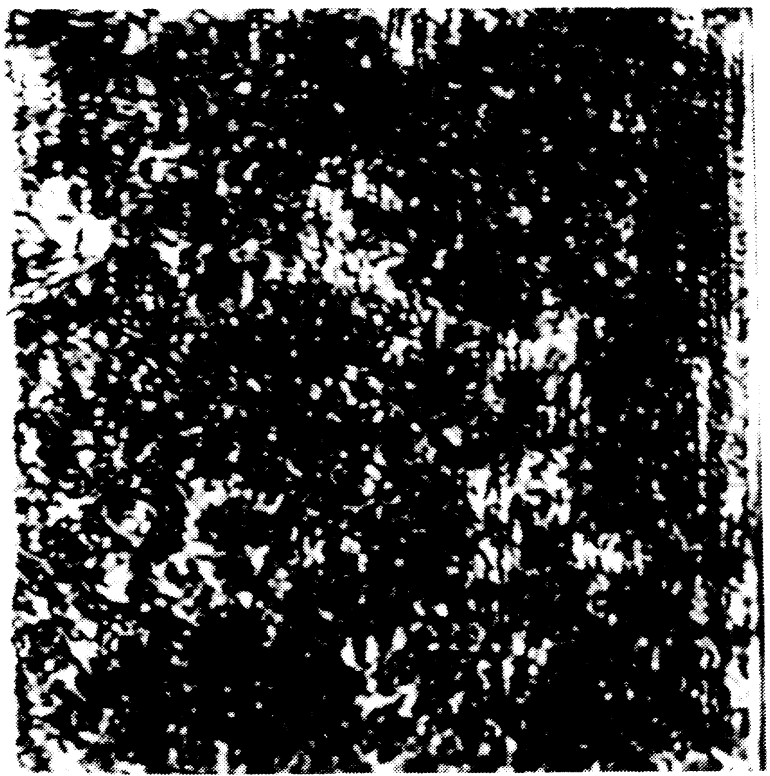

b

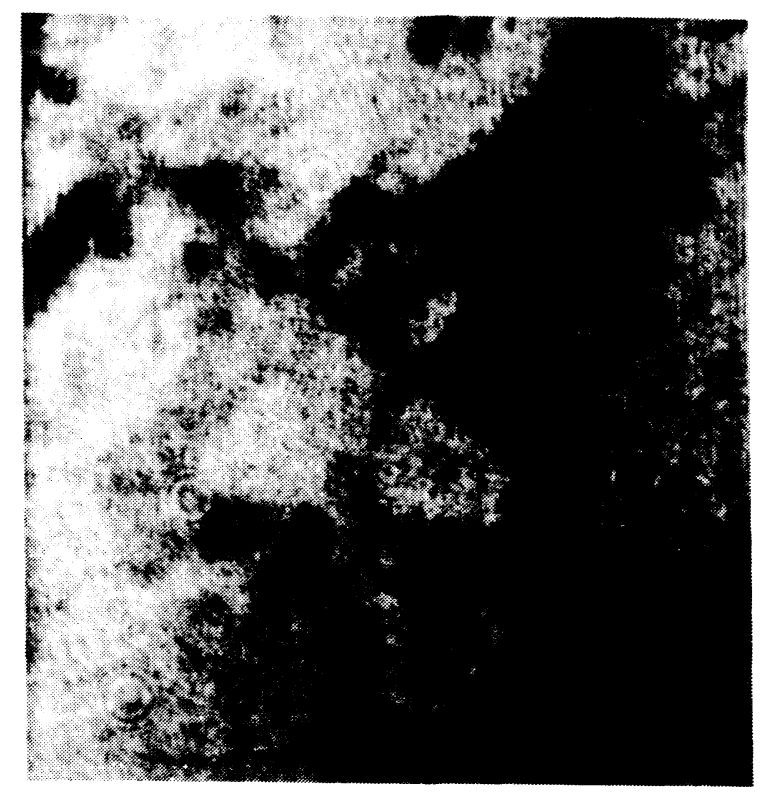

Figure 3

Scene used in optical processing from matrix camera with raster (a). low-pass filtered with raster removal (b). and high-pass filtered (c). 
The middle-pass image appears to be a coarse copy of the original. with emphasis on vegetation areas (photo interpretation). figure $1 \mathrm{c}$. The high-pass image, with the filter passing only extreme high orders in the frequency domain. consists of noise. figure 1d. Allowing lower frequency domain orders in the high-pass filter can of course highlight edges of scene features.

For the filtering described above the high-and low-pass filters were applied between the FFT 2 and IFFT 2 algorithms. The middle pass process includes separating the real and imaginary components of the transform utilizing LAS/COMPSEP in the frequency domain. LAS/FILTER-MED was applied on the real image component in integer" 1 format. LAS/COMPLEX was then used to regenerate the complex transform. LAS/IFFT2 applied in two dimensions was used to regenerate the middle-pass filtered image.

The object for the optical processing experiment was the $35 \mathrm{~mm}$ negative of the TM scene taken from the matrix plotter. Figure $2 b$ shows the Fourier transform in the frequency domain for the optical processing. The series of low spatial frequency diffraction orders are due to the raster lines in the digital display unit from which the image is derived. Careful examination of the Fourier transform reveals that each raster order has a finite width orthogonal to the line of raster diffraction orders. These narrow horizontal lines are due to overlapping diffraction orders for the scan lines from the original image which are orthogonal to the raster lines. As the raster lines are of higher spatial frequency than the scan lines in the original digital image. the Fourier transform of the raster lines in the frequency domain have a significantly lower spatial frequency than the digital scan lines. This allows the scan lines to be filtered out of the final image. However, the digital image lines can create an interference effect that hides image features.

Figure $3 \mathrm{a}$ shows the image obtained with no filter in the optical processing. Figure $3 \mathrm{~b}$ shows the image filtered for low-pass with the filter size set to exclude the higher frequency domain orders for the raster pattern. The low-pass filter removes the raster scan lines while leaving the scene. Figure $3 \mathrm{c}$ shows the image filtered for high-pass leaving only noise. in agreement with figure 1d. While figures 1. 2 and 3 show only modest and well established results. they are useful for the students early understanding of the two processes. They demonstrate the complementary nature of the optical and digital processes. 
The results achieved by optical and digital image processing illustrated in this paper demonstrate parallel Fourier domain processing techniques. The filter designs in the two image processing sequences were analogous but were not applied with the same precision as to band pass filtering. This is partly due to the differences in the band pass filtering in the two techniques were developed. i.e. digital numerical matrix filtering versus physical component filtering. The other part resulted as an illustration component. as raster lines were added to the negative image to illustrate a system involving film raster capture from a crt/matrix camera type device that might be used to record image data. The raster lines then occurred in the film in addition to the scan lines generated by the radiometrically uncorrected version of Thematic Mapper (TM) data furnished by NASA/GSFC for the experiment. The TM data has several differing periodic responses due to detector system and voltage variability occurring in the instrument. ${ }^{14}$ This provided additional periodicities for sinusoidal variations expressed in the transforms of the data (see figure 2a.) As the objective of the paper was to illustrate similar parallel Fourier domain processing sequences. the sources of variability were not investigated but assumed to be related to the above mentioned sources. The information and data available was insufficient to provide these correlations.

The author's took advantage of the periodicity in the data to illustrate some of these features of band pass filtering in the digital and optical Fourier domain. While some attempts were made to correlate the features expressed by the two processes. the main objective is met by the illustration of techniques initialized in digital and Optical Fourier domain image processing

Note that the above discussions relate to specific hardware and software capabilities at Marshall University. However. if the digital image processing is not available on this sophisticated level a one-dimensional FFT program can still be used to make analogies with the optical domain. In particular. the removal of raster patterns can be approached as a one-dimensional exercise between spatial and frequency domains. Thus, an optical system and access to a relatively simple FFT program can be used to illustrate the same principles.

Optical filtering and digital electronic filtering using Fourier transforms are complementary processes. Teaching the digital process has better results if the students use the optical process as a visualization tool. Teaching the optical process benefits from students having access to the digital process in order that students see the degree of control and accuracy available in the digital domain. Analogies between the two processes can be used to illustrate and reinforce physical principles. This process can be extended to a subject area such of audio sound reproduction where discussions of high frequency components present in a complex sound wave contribute to the sharpness of a sound impulse. Similarly. high frequency components. illustrated with high-pass filters. in a digital or optical domain can be used to illustrate enhancement of edge features in a two-dimensional image. Extending the logic to an industrial setting. machine-vision can be used to locate objects by filtering out low frequencies from the 
Fourier transform domain to enhance object edges. The enhanced edges in turn locate the object for the machine.

Teaching courses in optics. electronics. and digital image processing over a number of years has resulted in a multi-disciplinary approach to problem solving in all of the topics. Students can understand applications, such as machine-vision. better after observing physical principles in the optical and/or digital domains first. It is believed. by the authors. that the presentation of each topic is strengthened by the introduction of analogies from the other topics.

\section{ACKNOWLEDGMENTS}

The authors gratefully acknowledge the generous support of the West Virginia Legislature. especially the Speaker of the House. Charles "Chuck" Chambers. and the President of the Senate. Keith Burdette. They coordinated for the donation and support of the two MicroVAX II computers and the I2S image display system. in behalf of the West Virginia Legislature. This was achieved with support and cooperation from the Digital Equipment Corporation.

In addition. this work was supported in part by Dr. Yin-Chi Lu and Dr. Philip Pease of NASA/Goddard Space Flight Center who generously donated machine-time and expertise for the electronic image processing before the equipment at Marshall was functional in the new facilities. The authors gratefully acknowledge this support with a system compatible with the Marshall University system.

\section{REFERENCES}

I. Ralph Oberly and James O. Brumfield. "Flexible New Optics Facilities with Electronics and Computer Systems Integration in a Renovated Building at a Smaller State University," SPIE Proceedings, Education in Optics, Leningrad, 1991.

2. J. W. Goodman, Introduction to Fourier Optics, McGraw-Hill, New York. 1968.

3. Rafael C. Gonzalez and Paul Wintz. Digital Image Processing. Addison-Wesley, Reading, 1987.

4. Martin D. Levine. Vision in Man and Machine. McGraw-Hill. New York, 1985.

5. NASA/Goddard Space Flight Center, Land Analysis System Manual (LAS), Greenbelt, Maryland, 1989. 
6. J. O. Brumfield, A. Miller, V. Robinson and A. Yost, "EarthNET: Spatially Distributed Processing Experiment for Earth Resources Data," paper presented at the International Conference on Advanced Technology for Monitoring and Processing. Global Environment Information. London, September 9-13, 1985.

7. J. O. Brumfield, and H. H. L. Bloemer, "Global Distributive Computer Processing Systems for Environmental Monitoring. Analysis and Trend Modeling in Early Warning and Natural Disaster Mitigation," presented to the International Society of Photogrammetry and Remote Sensing, Commission VII. Enschede. August 25-29, 1986.

8. Alan J. Yost and J. O. Brumfield, "Integrated Image Processing and Geographic Information System Using Distributed Parallel Processing in Cartographic Operation," Proceedings of the Willi Nordberg Symposium of 1987, Remote Sensing: Towards Operational Cartographic Application, Graz, September 7-9, 1987.

9. J. O. Brumfield, Ralph E. Oberly and H. H. L. Bloemer, "Hardware Systems Design in Geobiophysical Modeling for Global Change," Proceedings of the International Geographic Information Systems Symposium '89, E. H. Pechan and Associates, pp 147-156, Baltimore, March 18 \& 19, 1989.

10. J. O. Brumfield, H. H. L. Bloemer, and Ralph E. Oberly, "Software Systems Design in Geobiophysical Modeling for Global Change," Proceedings of the International Geographic Information Systems Symposium '89, E. H. Pechan and Associates, pp 137-146, Baltimore, arch $18 \& 19,1989$.

11. George O. Reynolds, John B. DeVelis. George B. Parrent. Jr., and Brian J. Thompson, The New Physical Optics Notebook: Tutorials in Fourier Optics. Chapter 30. SPIE Optical Engineering Press, Bellingham, 1989.

12. Hugh H. Kieffer, Eric M. Eliason, Pat S. Chavez, Jr., "Intraband Radiometric Performance of the LANDSAT-4 Thematic Mapper," LANDSAT-4 Science Investigations Summary, John Barker, Editor, Volume II, pp 33.34, NASA Conference Publication 2326, Greenbelt, 1984.

13. Nilliam C. Likens and Robert C. Wrigley, "Impact of LANDSAT MSS Sensor Differences on Change Detection Analysis," LANDSAT-4 Science Investigations Summary, John Barker, Editor, Volume II, pp 87-90, NASA Conference Publication 2326, Greenbelt. 1984.

14. Michael D. Metzbrand, William A. Malila, "Scan - Aug 6 and Detector Effects in Thematic Mapper Radiometry," LANDSAT-4 Science Investigations Summary, John Barker, Editor, Volume II, pp 23-27, NASA Conference Publication 2326. Greenbelt, 1984. 